\title{
Polymodal roles of transient receptor potential channels in the control of ocular function
}

\author{
Peter S Reinach ${ }^{1 *}$, Weiwei Chen ${ }^{1}$ and Stefan Mergler $^{2}$
}

\begin{abstract}
Maintenance of intracellular $\mathrm{Ca}^{2+}$ levels at orders of magnitude below those in the extracellular environment is a requisite for preserving cell viability. Membrane channels contribute to such control through modulating their time-dependent opening and closing behaviour. Such regulation requires $\mathrm{Ca}^{2+}$ to serve as a second messenger mediating receptor control of numerous life-sustaining responses. Transient receptor potential (TRP) channels signal transduce a wide variety of different sensory stimuli to induce responses modulating cellular function. These channels are non-selective cation channels with variable $\mathrm{Ca}^{2+}$ selectivity having extensive sequence homology. They constitute a superfamily made up of 28 different members that are subdivided into 7 different subfamilies based on differences in sequence homology. Some of these TRP channel isotypes are expressed in the eye and localized to both neuronal and non-neuronal cell membranes. Their activation generates intracellular $\mathrm{Ca}^{2+}$ transients and other downstream-linked signalling events that affect numerous responses required for visual function. As there is an association between changes in functional TRP expression in various ocular diseases, there are efforts underway to determine if these channels can be used as drug targets to reverse declines in ocular function. We review here our current knowledge about the expression, function and regulation of TRPs in different eye tissues in health and disease. Furthermore, some of the remaining hurdles are described to developing safe and efficacious TRP channel modulators for use in a clinical setting.
\end{abstract}

Keywords: Transient receptor potential ion channels, Calcium, Retina, Cornea, Uvea, Lens

\section{Introduction}

\section{TRP channel relevance to ocular function}

One of the reasons why ocular transient receptor potential (TRP) channels are essential for maintaining normal vision is that some of their subtypes transduce environmental stresses into cell signalling events to control different physiological responses that alter cellular function. This realization has prompted intense research efforts to more extensively characterize how they control responses countering losses in cellular function caused by tissue injury, touch, fluctuations in ambient temperature, $\mathrm{pH}$ as well as osmolarity, hormonal exposure and pathogen tissue infiltration. Such insights can lead to the identification of lead compounds whose development may provide novel agents for improving the treatment of different ocular diseases in a clinical setting.

Functional TRP expression has been identified in every ocular tissue eliciting responses to a host of sensory

\footnotetext{
* Correspondence: preinach@yahoo.com

'School of Ophthalmology and Optometry, Wenzhou Medical University, Wenzhou, Zhejiang 325027, P.R. China

Full list of author information is available at the end of the article
}

stimuli under both physiological and pathophysiological conditions. By virtue of their non-selective cation permeability, their activation elicits intracellular $\mathrm{Ca}^{2+}$ transients ultimately triggering pain, scarring and inflammation to these various environmental activators [1,2]. There is heightening interest by drug companies in screening pipeline compounds targeting different ocular TRP channel subtypes because some of them are promising targets for reversing pathophysiological changes that compromise ocular function. For example, in different animal models of hypertensive glaucoma and injury-induced cornea scarring, declines in TRP function are associated with reductions in excitotoxic-induced retinal ganglion cell (RGC) dysfunction and corneal stromal fibrosis [3-5]. On the other hand, implying that suppressing TRP activation has therapeutic potential in glaucoma may be premature because of a concern about this model's physiological relevance [1]. Another concern regarding the role of changes in functional TRP expression to visual processing stems from a more recent study showing that loss of TRP function instead inhibits optic nerve function [6]. These contradictory findings show that it is still unclear 
whether developing either novel TRP agonists or antagonists is the preferred approach for effectively treating glaucoma. This caveat prompts studies to unravel complexities of TRP channel involvement in ocular function. To shed some light on this complex question, we review literature describing ocular TRP channel functional expression in the cornea, conjunctival epithelium, uvea, lens and retina. In addition, the possible association between TRP channel mutations and some ocular diseases is considered.

\section{Review}

\section{TRP channel nomenclature and channelopathies}

Studies on Drosophila retinal phototransduction were the driving force for subsequent work on TRP channels. In the initial report, a mutant blind strain was identified in which a light flash elicited an aberrant transient rather than sustained retinal membrane depolarization [7]. Subsequently, it was realized that the channel eliciting this altered response has a homology with TRP channels. The TRP prototype was cDNA cloned from sensory neurons and functionally expressed nearly 30 years later [8]. This cloned channel is the forerunner of all subsequently identified TRP isoforms. Early on, it was referred to as the capsaicin receptor since this red pepper extract constituent is an agonist of this channel. This prototype has sequence homology with 28 different subtypes that together constitute a TRP superfamily $[9,10]$. In the TRP classification scheme, it is designated as TRP vanilloid 1 (TRPV1). All superfamily members assemble as tetramers and have a common configuration: six transmembrane spanning units in which the span between the fifth and sixth segments forms a conduit for cation membrane permeation. This superfamily is separated into seven different subfamilies and includes: a) TRPA (ankyrin); b) TRPC1-4 (canonical); c) TRPM 1-8 (melastatin); d) TRPML (mucolipin); e) TRPN (no mechanopotential); f) TRPP (polycystin); g) TRPV1-7 (vanilloid) [11]. Drosophila phototransduction is mediated by a TRPC channel together with a second TRPC channel, trp-like (TRPL) [12]. The relevance of defining TRP channel subtypes and their function is now more apparent since changes in their protein expression and function underlie either many hereditary diseases (so-called TRP channelopathies) or contribute indirectly to the genesis of several diseases including cancer [13-15].

\section{TRP activation overview}

TRP channels are cellular sensors involved in the formation of sight, hearing, touch, smell, taste, temperature, and pain sensation. These diverse TRP functions are elicited through activation of downstream cell signalling pathways and allow the host cell to respond to benign or harmful environmental changes. The molecular mechanisms underlying TRP channel activation are very complex and not yet fully understood. TRP activation is caused by an increase in channel open time, which can be prolonged by a host of different factors. For example, changes in membrane stretch and exposure to an anisosmotic challenge (such as TRPV1 and TRPV4) as well as kinase-induced channel phosphorylation all lead to TRP activation whereas cooling directly can activate TRPM8 [16-18]. Another type of activation of TRPs occurs via more or less specific ligands. Initially, it was realized that either exogenous small organic synthetic compounds or natural products could activate TRPs. Even though TRPV1 was originally designated as the capsaicin receptor, subsequent studies revealed that it also interacts with other TRP isoforms. This red pepper extract constituent also activates voltage-gated inward and outward currents as well as impairs mitochondrial function and inhibits prostaglandin E2 production through non TRPV1 pathways [1]. There are indications in the retina that it may also activate TRPM1 channels. In addition, there may be other sites of interaction since in TRPM1 ${ }^{-1-}$ mice; the responses to capsaicin were not fully attenuated. Other (synthetic) agonists are icilin (TRPM8), which was also described as a super cooling agent [19] or camphor (TRPV3) [20,21]. Furthermore, endogenous lipids or products of lipid metabolism are also ligands of TRPs. Finally, inorganic ions such as $\mathrm{Ca}^{2+}$ or $\mathrm{Mg}^{2+}$ can also (directly) activate TRPs (e.g. TRPM6 for $\mathrm{Mg}^{2+}$ [22] or TRPA1 for $\mathrm{Ca}^{2+}$ [23]). However, it is not yet known if the TRP conformational changes induced by $\mathrm{Ca}^{2+}$ or $\mathrm{Mg}^{2+}$ are the same as those induced by a thermal transition known to activate these different TRP channel subtypes.

Overall, classical activation of TRPs and in particular thermo-TRPs are mostly related to direct activation via mechanical stimuli or channel phosphorylation or certain exogenous and endogenous agents or inorganic ions. Given the complexities in identifying selective TRP channel modifiers, any drug target assignment should be verified by determining if a drug-induced response can be mimicked in transgenic mice having either a selective TRP gain or loss of function. If a drug effect cannot be replicated, it is possible that it will instead be caused by a compensatory response provided by modulation of another TRP subtype leaving drug selectivity uncertain.

\section{Reciprocal TRP channel and G-protein coupled receptor activation}

G-protein-coupled receptors (GPCRs) and TRP channels are coexpressed on sensory neurons and act as sensors of noxious irritants and inflammatory stimulants. With approximately $850 \mathrm{GPCR}$ variants in mammals, they are the largest family of signalling proteins. Due to their responsiveness to the largest group of diverse structural ligands, $30 \%$ of current drugs target GPCRs to treat pathophysiological conditions. More than 40 GPCR 
families contribute to pain processing and function as sensors on peripheral nerves to detect noxious, irritant, and inflammatory stimuli, including proteases, peptides, amines, and lipids [24]. Sensory nerves express TRP channels, which directly sense endogenous and exogenous chemical, mechanical, and thermal stimuli. These nonselective cation channels are also major downstream effectors of GPCR signalling. This GPCR-TRP axis is responsible for eliciting pain, itch, cough, and neurogenic inflammation [25-27]. Signalling pathways that emanate from the GPCR superfamily lead to altered TRP channel activity or expression. GPCR signalling can generate mediators that stimulate TRP channels (i.e., channel activation) or instead enhance their responses to TRP agonists (i.e., TRP sensitization). For example, TRPV1 activity can be modulated by a wide variety of endogenous small molecules through different regulatory mechanisms that either increase or decrease its sensitivity to different conditions known to either stimulate or inhibit this channel. In one case, TRP channels can amplify GPCR effects and mediate their contributions to transmission of pain, itch, cough, and neurogenic inflammation. Conversely, GPCR activation can generate mediators that stimulate TRP channels (i.e., channel activation) or that enhance their responses to TRP agonists (i.e., TRP sensitization). The net result is that TRP channels can amplify the effects of GPCRs and mediate their contributions to transmission of pain, itch, cough, and neurogenic inflammation [28].

\section{Dependence of TRP channel-induced phototransduction on GPCR activation}

The mutant channel accounting for the aforementioned aberrant phototransduction response in the blind Drosophila strain was found in later studies to have a socalled TRP box structural sequence. This sequence is common to all TRPs including TRPV1, the founding member of the TRP superfamily. This 28-member superfamily forms the basis of receptor-operated TRP channel gating across species. In the plasma membrane of Drosophila photoreceptors, the receptor-operated channel activity requires GPCR activation and release of a heterotrimeric GTP bound G-protein complex, phospholipase $\mathrm{C}$ activity, and metabolism of phospholipids and phosphotidyl inositol-4,5 bisphosphate ( $\left.\mathrm{PIP}_{2}\right)$ leading to the generation of inositol trisphosphate $\left(\mathrm{IP}_{3}\right)$, membraneassociated diacylglycerol (DAG) and arachidonic acid. $\mathrm{IP}_{3}$ binds to its receptor on intracellular stores to release $\mathrm{Ca}^{2+}$ from intracellular stores (ICS) and DAG stimulates kinase activity. $\mathrm{Ca}^{2+}$ losses leading to the activation of storeoperated $\mathrm{Ca}^{2+}$ channels (SOCs) are composed of TRP subunits such as TRPC1 and TRPC4 [29]. SOC activation results in the refilling of the depleted ICS. Although the mechanism is still not clear, the activation threshold for TRP channel activation is lowered (i.e. sensitized) by the influx of $\mathrm{Ca}^{2+}$ and fatty acid second messengers to permit channel opening leading to light-induced current and membrane depolarization. This process is desensitized by DAG-sensitive kinase phosphorylation of the TRP channel and the $\mathrm{Ca}^{2+}$ sensitive protein calmodulin (CaM), which binds to the channel preventing channel reactivation [30].

\section{Thermosensitive TRP channel activation}

The ability to sense environmental temperatures and to avoid noxious heat or cold is crucial for the survival of all organisms. Six different thermosensitive TRP isotypes provide such protection in different ocular tissues. For TRPV1-4 [31] and TRPM8/TRPA1 [32-34] their activity is temperature sensitive within specific ranges. TRPV1-4 as well as TRPM8 and TRPA1 sensitivities span almost the entire temperature range to which mammals are exposed. In the vanilloid subfamily, TRPV 4 and TRPV3 are activated by temperatures from 25 to $31^{\circ} \mathrm{C}$, respectively, whereas TRPV1 is activated at $43^{\circ} \mathrm{C}$ and TRPV2 at a noxious temperature of $51^{\circ} \mathrm{C}$ [35]. On the other hand, TRPM 8 and TRPA1 are activated below 25 and $17^{\circ} \mathrm{C}$, respectively. The mechanism(s) underlying these different temperature sensitivities are poorly understood.

Three hypothetical mechanisms may account for the origin of these remarkably steep temperature sensitivities. They include: 1) steep specific temperature dependence for ligand binding; 2) temperature-induced channel rearrangement; 3) temperature-dependent membrane tension changes may lead to channel opening [31]. A recent report suggests that differences in TRP channel protein sequence endow thermal sensitivity, which could mean that this property is ascribable to specific structural changes. This suggestion stems from the finding that in the Drosophila TRPA1 isoform, thermal sensitivity variability is linked to changes in the expression of a specific 37-amino-acid long intracellular region (encoded by a single exon) [36]. TRPM8 stimulation could result in graded shifts of voltage-dependent activation to more negative membrane voltages [37]. This channel may be also regulated by GPCRs. One indication for such an interaction is that both activation of a GPCR and a nerve growth factor receptor inhibited menthol- and coldinduced TRPM8 activity [38]. Using temperature sensitivity as a criterion for channel identification may be more reliable than relying on drug sensitivity due to uncertainties of drug selectivity. Another reason for assessing changes in thermosensitive TRP channel expression is that they may underlie sensitivity differences in sensory neurons to cold and mechanical stretch [39]. Such a possibility has prompted interest in developing drugs targeting thermosensitive TRP expression since they may prove to be useful antinociceptives. 
TRPs in ocular tissues and cells

\section{Retina, pigment epithelium and uvea}

Retinal TRPV1 gene transcripts and protein expression have been identified in species ranging from teleosts, amphibians, birds to mammals. Even though capsaicin is routinely used to characterize functional TRPV1 expression, there is uncertainty about its selectivity since it has side effects. Capsaicin may also activate a TRP melastatin receptor (i.e. TRPM1) described in the retina [40]. However, this is questionable since capsaicin also elicited current responses in $\mathrm{TRPM}^{-/-}$mice [41]. A recent report showed that the capsaicin response is eliminated in mice lacking the mGluR6 receptor. This suggests that this agonist may also act on either this metabotropic receptor or its downstream GTP binding protein $\left(G_{0}\right)$ heteromers [42]. The caveat of sole reliance on capsaicin effects to identify TRPV1 activity also applies to capsazepine (CPZ), which is commonly used as a TRPV1 antagonist. This cautionary note is indicated since $\mathrm{CPZ}$ also has effects in TRPV1 ${ }^{-/-}$mice. These responses include antagonism of voltage-gated $\mathrm{Ca}^{2+}$ channels, acetylcholine receptors, hyperpolarization-activated cation channels, and stimulation of amiloride-sensitive ENaCs [1]. Another cautionary note is indicated regarding protein expression since with different antibodies and protocols, the number of reported bands is inconsistent. This variability points to the need to validate all results by showing negative staining in Western blots in fractions obtained from TRPV1 ${ }^{-1-}$ mice. Nevertheless, TRPV1 expression may be involved in controlling ontogenetic development. It may regulate the synaptic plasticity during superior colliculus development [1]. Despite these uncertainties, maximal activation of TRPV1 by capsaicin has been shown to induce neuronal and glial excitotoxicity in some areas of the brain and apoptosis in cultured RGCs. Based on some studies suggesting that TRPV1 is mechanosensitive, Sappington et al. determined if TRPV1 is a target for RGC death in vivo during exposure to elevated intraocular pressure (IOP). As raising the pressure in an isolation chamber by $70 \mathrm{mmHg}$ caused increased RGC death, the authors surmised that elevated intracellular $\mathrm{Ca}^{2+}$ levels were attributable to neurotoxicity caused by TRPV1 hyperactivation [3]. These data provide an interesting retinal disease model for RGC remodelling and apoptosis elicited by increases in intracellular $\mathrm{Ca}^{2+}$ levels during exposure to elevated pressures that induce mechanosensitive TRPV1 hyperactivation.

Even though TRPV1 hyperactivation may be deleterious under some conditions, there are contradicting reports showing that this change is instead neuroprotective in other contexts. Specifically, in TRPV1 ${ }^{-/-}$mice, elevated IOP was more damaging to RGCs when compared to the wildtype counterparts, suggesting that TRPV1 expression is instead neuroprotective [6]. Furthermore, in CPZ-treated rats, anterograde retrograde transport of markers were inhibited, which was associated with axonal loss and astrogliosis. These findings prompted suggestions that TRPV1 expression is instead neuroprotective during RGC exposure to retinal stress. Taken together, these opposing effects of alleged changes in TRPV1 activity may be context dependent.

Various other TRPs are also detected in the mouse retina (TRPC1-4 TRPM1/3/7, TRPML1, TRPP2, TRPV2, TRPV4 $[43,44]$. In mouse RGCs, TRPV4 modulates calcium flux, spiking rate, and apoptosis. Furthermore, TRPC1 and TRPC4 are expressed in the chicken retina [45]. TRPM1 is expressed on ON-bipolar cell dendrites that invaginate photoreceptor terminals and on the synaptic ribbons of a subclass of rods. This expression pattern suggests a dual function for TRPM1 in the ONpathway [40]. TRPV1, TRPM8 and TRPA1 are expressed in retinal tumour cells (retinoblastoma). Interestingly, the expression of TRPA1 was completely suppressed in a retinoblastoma cell line, which is resistant to the cytostatic agent etoposide [46]. In addition, TRPV1-4, TRPM8 and TRPA1 expressions in retinal pigment epithelial (RPE) cells are described in a review describing ocular tissue specific gene expression patterns [47]. Increasing the ambient temperature or insulin-like growth factor-1 (IGF-1) increased the vascular endothelial growth factor-A (VEGF-A) secretion rate in RPE cells indicating that TRPs are involved in the IGF-1 induced response [48]. Regarding the human uvea, there is only one study demonstrating gene expressions for TRPV1, TRPM8 and TRPA1. Notably, in human uveal melanoma cells, the gene expression of TRPM8 is at a lower level whereas the TRPA1 expression is at a high level in healthy uvea [49].

\section{Conjuncitva and limbus}

There are functional thermosensitive TRPM8, TRPV1 and TRPV2 as well as TRPV4 expressions in conjunctival epithelial cells [50,51]. Functional TRPM8 expression is evident based on increases in intracellular $\mathrm{Ca}^{2+}$ levels and currents elicited by icilin that were blocked by the TRPM8 antagonist, BCTC. Similarly, BCTC also blocked these increases induced by a thyroxin metabolite, thyronamine (T1AM), indicating that it is an endogenous TRPM8 agonist. TRPM8 activation by either icilin or T1AM suppressed TRPV1-induced increases in interleukin 6 (IL-6) expression indicating intracellular signalling interaction or crosstalk between these TRP channels. TRPV1 expression is functional since capsaicin-induced $\mathrm{Ca}^{2+}$ transients, ionic currents and increases in proinflammatory IL-6 release were all blocked during exposure to CPZ. TRPM8 and TRPV1 coexpression in these cells as well as on afferent trigeminal ophthalmic branch sensory neurons is supportive of the 
notion that both of these receptors contribute to the maintenance of ocular surface health [52-55]. Cold stimulation of TRPM8 increases in mice lacrimation whereas TRPV1 activation by noxious agents induces pain and avoidance behaviour [56,57]. On the other hand, the TRPV1-induced increase in proinflammatory cytokine release occurring in dry eye disease (DED) may be moderated through receptor crosstalk between TRPM8 and TRPV1 [50]. Therefore, it may be advantageous to evaluate the effectiveness of TRPM8 agonists to increase lacrimation and reduce TRPV1-induced immune responses.

\section{Corneal epithelium}

In rats, mice and human corneal epithelia, there are TRPV1-4 expressions [58-60]. Their presence suggests that this tissue layer is well poised to counter challenges to its functional competence caused by exposure to noxious agents, anisosmotic challenges, elevated temperatures and pathogens. Furthermore, the mitogenic response to epidermal growth factor receptor (EGFR) phosphorylation by EGF is dependent on increases in plasma membrane $\mathrm{Ca}^{2+}$ influx elicited by a SOC composed of TRPC4 subunits. This dependence is evident since knockdown of TRPC4 expression in human corneal epithelial cells (HCEC) markedly reduced cell proliferation and migration [61]. Conversely, another important TRPV1 function is that its activation by capsaicin induces increases in cell proliferation and migration through transactivating EGFR [62]. The HCEC mitogenic response to capsaicin is consistent with a mouse study in which corneal reepithelialization following debridement was delayed in TRPV1 $^{-1-}$ knockout mice compared to their wildtype counterparts. This stimulatory effect of TRPV1 in promoting cell proliferation and migration is associated with increases in IL-6 and substance P expression, which are coactivators of growth factor induced wound healing [58]. TRPV4 expression was identified in intact human corneal epithelium and its activation by exposure to a hypotonic challenge is required for inducing regulatory volume decrease behaviour [63]. Furthermore, hypertonic stresses identical to those described in some dry eye patient tears elicited through TRPV1 channel activation, raised proinflammatory cytokine levels by eliciting mitogen-activated protein kinase (MAPK) and nuclear factor- $\mathrm{kB}$ (NF-kB) activation $[64,65]$. Taken together, corneal epithelial functional TRP expression contributes to countering anisosmotic challenges similar to those identified in the tears of some dry eye patients. Such stress can compromise maintenance of corneal epithelial barrier function rendering the tissue layers vulnerable to pathogenic infiltration and declines in transparency.

\section{Corneal stroma}

Subsequent to the breaching of the corneal epithelial basement membrane by a severe injury, transforming growth factor $-\beta$ (TGF $\beta$ ) infiltrates into the stroma and activates its cognate receptor on human fibroblasts also expressing TRPV1. These two receptors interact through crosstalk to induce myofibroblast transdifferentiation leading to fibrosis and corneal opacification subsequent to a severe injury [66]. This dependence on TRPV1 activation is evident since during exposure to $\mathrm{CPZ}$, exogenous TGF $\beta-1$ failed to elicit increases in $\alpha$-smooth muscle actin (SMA) expression. This result is pertinent for efforts to reduce corneal opacification and inflammation following a chemical burn since it suggests that in a clinical setting treatment, a TRPV1 antagonist could promote restoration of corneal transparency by inhibiting TGF $\beta$-induced myofibroblast transdifferentiation. It is noteworthy that loss of TRPV1 function in two different mouse wound healing models has opposite effects on restoring transparency during corneal wound healing. In the epithelial layer, wound closure is delayed due to declines in cell proliferation and migration whereas in the stroma, fibrosis and inflammation are suppressed. These polymodal effects of TRPV1 activation suggest that TRPV1 antagonist usage in a clinical setting may need to be restricted to cases involving penetrating stromal injury in which case corneal opacification occurs due to fibrosis and dysregulated chronic inflammation [67].

\section{Corneal nerve fibres}

TRPA1 and TRPM8 are expressed on corneal afferent nerves. Corneal nerve fibres are radially distributed within the stroma and the adjacent limbus [68]. Mouse, guinea pig and human corneal nerve fibres contain sensory neurons in which TRPV1 and TRPM8 are highly expressed at levels similar to those in their non-corneal counterparts $[54,55,57]$ and in non-corneal primary sensory neurons [35]. These channels mediate excitation and subsequent desensitization to capsaicin (TRPV1) or menthol (TRPM8). In a clinical context, thermosensitive TRPM8 function in nerve fibres may be relevant for dry eye syndrome treatment since corneal cooling below $23^{\circ} \mathrm{C}$ increased lacrimation through TRPM8 activation whereas warming had an opposite effect [57].

\section{Corneal endothelium}

Rae and Watsky [69] first described that in the corneal endothelium, whole-cell configuration of patch-clamp recordings showed an outwardly rectifying thermosensitive current along with a $\mathrm{K}^{+}$-selective current. The origin of this thermosensitive component was not identified but may be attributable to TRP activity [69]. More recently, thermosensitve TRPV1-4 channel activities were identified in human corneal endothelial cells [70-72]. 
However, the functional importance of their presence is still unclear. Thermosensitive TRPM8 was identified in human corneal endothelial cells based on showing that application of the super cooling TRPM8 agonist icilin reversibly increased intracellular $\mathrm{Ca}^{2+}$. One possible upshot of TRPM8 presence in the endothelium is that preservation of its function in an eye bank setting may be dependent on its activation during storage at temperatures below $23^{\circ} \mathrm{C}[73,74]$. Such expression may contribute to the ability of the endothelial cell layer to maintain tissue homeostasis function over a broader temperature range. Besides TRPM8 expression in human corneal endothelial cells, there might also be other TRPs expressed in human corneal endothelium since $\mathrm{H}_{2} \mathrm{O}_{2}$ induced significant increase in $\left[\mathrm{Ca}^{2+}\right][75]$. These limited results indicate that there remains a large void in our understanding of the contribution made by functional TRP expression to the maintenance of corneal transparency and deturgescence.

\section{Lens epithelium}

Regarding lens (epithelial) cells, there is very limited information on the functional involvement of TRPs. TRPV4 in porcine lens epithelium regulates hemi-channel-mediated ATP release and $\mathrm{Na}^{+}-\mathrm{K}^{+}$-ATPase activity [76]. In addition, TRPV1 gene expression was identified in the lens and its gene expression is at a very high level compared to that in the retina [77].

\section{TRP channelopathies and ocular disease}

TRP gene mutations underlie numerous inherited diseases in humans including the eye. These diseases are associated with dysfunctional channel expression and are termed TRP channelopathies [13,14,78]. So far, TRP channelopathies have been linked to cardiovascular, renal, skeletal and nervous system pathologies [13]. With respect to the eye, there are only a few such studies. One of them deals with mucolipidosis type IV (MLIV). It is an autosomal recessive, neurodegenerative lysosomal storage disorder, which is due to mutations in the gene MCOLN1. MLIV is clinically characterized not only by ophthalmologic abnormalities such as corneal opacity, retinal degeneration and strabismus, but also by other nonocular abnormalities. MCOLN1 which encodes the protein mucolipin 1 (MNL1) is a non-specific cation channel (TRPML1) [79]. In addition, human TRPM1 mutations are associated with congenital stationary night blindness (CSNB), whose patients lack rod function and suffer from night blindness starting in early childhood [80].

\section{Dry eye syndrome}

At this point, it is unclear whether there is an association between a TRP channelopathy and DED. Nevertheless, even without a mutated expression, an altered level of expression may trigger or promote DED. Based on current evidence, there are indications that either one or both of the aforementioned changes could contribute to DED. In DED, ocular surface inflammation and desiccation may stem from dysfunctional TRPV1 and TRPM8 expressions and/or conditions in this disease that promote their hyperactivations. With regards to TRPV1, it is activated by hypertonic conditions similar to those identified in some DED afflicted patients. In vitro, such levels lead to increases in the expression of the same proinflammatory cytokines, chemokines and matrix metalloproteinases, whose levels are elevated in patient tears. TRPM8 is also implicated since its expression on afferent sensory corneal nerves and in the cell layers contributes to maintenance of basal tear flow during thermal stress $[51,57,64]$. Taken together, TRPM8 agonists should be evaluated in various dry eye animal models to determine if they reduce dry eye symptomology and dampen TRPV1-induced inflammation and corneal opacification.

\section{Diabetic retinopathy}

In diabetic retinopathy, impaired blood flow due to vascular occlusion can lead to retinal hypoxia causing photoreceptor and neuronal cell death and visual impairment. There are various studies describing a connection between TRPs and diabetes mellitus $[81,82]$, but none directly relates to diabetic retinopathy. On the other hand, some are available pertaining to non-diabetic related retinopathy (e.g. melanoma-associated retinopathy) $[80,83,84]$.

\section{Glaucoma}

One of the pathological events in this insidious disease includes declines in RGC function due to IOP elevations compressing nerve fibres traversing through the optic nerve head. Changes in TRP function may contribute to changes in RGC function induced by such stress since they are expressed in this tissue [3,41]. Other tissue changes that may contribute to glaucoma damage are obstruction of aqueous outflow pathways through the trabecular meshwork (TM) and/or a change in ciliary muscle contractility. References describing TRPC channels in these tissues are listed in a recent review [45]. As their activities are sensitive to changes in intracellular $\mathrm{Ca}^{2+}$ content, they are referred to as SOCs. It has been suggested that 11 different mechanosensitive TRP channels identified in trabecular meshwork cells could be activated by increases in IOP and contribute to TM pathology [85]. Therefore, developing selective drug targeting mechanosensitive TRP channels may provide a novel option to treat hypertensive glaucoma.

\section{Cataract}

Increase in intracellular $\mathrm{Ca}^{2+}$ level is associated with cataractogenesis [86]. TRPV1 immunostaining was identified in 
the lens and its activation may contribute to cataractogenesis since such an effect induces rises in intracellular calcium [77]. A possible role is summarized in a review describing store-operated $\mathrm{Ca}^{2+}$ channels composed of TRPC subunit expressions (i.e. TRPC1 and TRPC5) in cataract development [47].

\section{Ocular tumours}

Ocular tumour incidence is rarer than in non-ocular tissues. Choroidal- or corneal tumorigenesis is unique to ocular tissues because it is not directly related to tumour neovascularization, which is a dangerous progression that promotes tumour growth and metastasis. Endothelial cell proliferation drives neovascularization and several different TRP subtypes have been detected in this tissue. However, there are no studies yet describing their contribution to tumour neovascularization.

\section{Uveal melanoma}

Uveal melanoma (UM) is a devastating disease. Once this tumour metastasizes out of the eye into the liver, lung, bone and skin, patient survival rates are poor. It is the second most prevalent malignant tumour of melanocytes $[87,88]$. This disease directly develops from degenerated melanocytes in the choroid. TRPM1 was designated as a melanoma metastasis suppressor based on its expression in normal pigment cells in the skin and the eye since it was absent in aggressive, metastasis-competent melanomas [89]. A similar association pertains to TRPA1 because its expression is lower in human UM cells than in healthy uvea [49]. An inverse relationship was described for TRPM8, which is expressed at higher levels in most of the investigated UM cell lines. Furthermore, TRPV1 and the cannabinoid receptor 1 (CB1) are functionally expressed in UM cells. Interestingly, activation of CB1 induced $\mathrm{Ca}^{2+}$ transients, which were suppressed by the TRP channel blockers $\mathrm{La}^{3+}$ and CPZ whereas capsaicininduced $\mathrm{Ca}^{2+}$ transients could also be suppressed by CB1 activation. Therefore, it is suggested that identification of functional TRPV1, TRPM8, TRPA1 and CB1 expressions in UM may provide novel drug targets for treatment of this aggressive neoplastic disease. A similar suggestion was made for treating non-uveal melanoma (TRPM8) [90].

Table 1 TRP channel pharmacology and ocular cell type localization

\begin{tabular}{|c|c|c|c|c|c|}
\hline TRP channel subtype & Compound & Concentration & Role & Expression/localization & References \\
\hline TRPC1 -TRPC3 & $2-A P B$ & $100 \mu \mathrm{M}$ & Antagonist & HCEC Mouse retina TM & {$[43,61,85]$} \\
\hline TRPC4 & $2-A P B$ & $100 \mu \mathrm{M}$ & Antagonist & RCECMouse retina & {$[43,61,93]$} \\
\hline TRPV1 & Capsaicin & $1-20 \mu \mathrm{M}$ & Agonist & $\begin{array}{l}\text { HCEC }{ }^{1} \text {, HCK HCEC-12 Rat retina HCjEC } \\
\text { Rat and mouse retinas Human retina } \\
\text { (tumour) Uvea (tumour) }\end{array}$ & {$[3,41,46,49,59,60,66,71,94]$} \\
\hline TRPV1 & Capsazepine & $1-10 \mu \mathrm{M}$ & Antagonist & HCEC HCK HCEC-12 HCjEC & {$[46,59,60,66,71]$} \\
\hline TRPV2 & $2-A P B$ & $200-400 \mu \mathrm{M}$ & Agonist & HCEC-12 HCEC & {$[71,95]$} \\
\hline TRPV2 & Camphor & $400 \mu \mathrm{M}$ & Agonist & RPE & [48] \\
\hline TRPV3 & $2-A P B$ & $200 \mu \mathrm{M}$ & Agonist & HCEC & [95] \\
\hline TRPV4 & $4 a-P D D$ & $5 \mu \mathrm{M}$ & Agonist & HCEC-12 HCEC ${ }^{2}, \mathrm{HCjEC}$ & {$[46,63,70]$} \\
\hline TRPV4 & GSK 1016790A & $5 \mu \mathrm{M}$ & Agonist & HCEC-12 & [70] \\
\hline TRPM8 & Menthol & $50-500 \mu \mathrm{M}$ & Agonist & HCNF HCEC-12 & {$[54,55,57,72,96]$} \\
\hline TRPM8 & Icilin & $2-60 \mu \mathrm{M}$ & Agonist & $\begin{array}{l}\mathrm{HCK}^{3}, \mathrm{HCEC}^{3} \mathrm{CEC}-12 \text { Human retina } \\
\text { (tumour) Uvea (tumour) }\end{array}$ & {$[46,49,72,75]$} \\
\hline TRPM8 & Eucalyptol & $3000 \mu \mathrm{M}$ & Agonist & HCEC-12 & [72] \\
\hline TRPM8 & $\mathrm{BCTC}$ & $10 \mu \mathrm{M}$ & Antagonist & HCEC-12 & [72] \\
\hline TRPA1 & Icilin & $2-60 \mu \mathrm{M}$ & Agonist & HCEC-12 TM & {$[72,75,85]$} \\
\hline
\end{tabular}

1activation by hypertonic challenge.

${ }^{2}$ activation by hypotonic challenge.

${ }^{3}$ Mergler et al. (unpublished data).

$\mathrm{HCEC}=$ human corneal epithelial cells.

RCEC $=$ rabbit corneal epithelial cells

$\mathrm{HCjEC}=$ human conjunctival epithelial cells.

$\mathrm{HCK}=$ human corneal keratocytes (stroma).

HCEC-12 = human corneal endothelial cells.

$\mathrm{HCNF}=$ human corneal nerve fibres.

$\mathrm{RPE}=$ retinal pigment epithelium.

$\mathrm{TM}=$ Trabecular meshwork. 


\section{Retinoblastoma}

Retinoblastoma (RB) is a malignant retinal tumour, which develops from immature retinal cells. Its incidence is low but is the most common ocular tumour of the eye in children and is associated with a RB mutation [91]. There have been only a few reports describing a TRP's involvement whereas other types of $\mathrm{Ca}^{2+}$ channels have been studied more extensively.

Regarding $\mathrm{Ca}^{2+}$ permeable TRPs, Hanano et al. suggested a possible significant regulatory role of TRPM7 for $\mathrm{RB}$ cell proliferation as a spontaneously activated $\mathrm{Ca}^{2+}$ influx pathway [92]. Another study revealed TRPV1, TRPM8 and TRPA1 gene expression in retinoblastoma cells. Notably, the expression of TRPA1 was suppressed in etoposide-resistant RB cells [46]. Therefore, using genetic approaches to upregulate TRPA1 expression could provide a means to induce etoposide sensitivity and suppress RB cell tumorigenesis.

Furthermore, CB1 receptors could be detected as shown in uveal melanoma cells. Activation of CB1 suppressed the TRPV1-induced $\mathrm{Ca}^{2+}$ increases in etoposidesensitive $\mathrm{RB}$ cells whereas this effect did not occur in etoposide-resistant RB cells. Nevertheless, the limited TRP characterizations suggest that there are clear differences between healthy and tumour ocular tissues or cytostatic-resistant ocular tissue. Therefore, modulation of TRP expression and/or function could provide a critically needed therapeutic option. In addition, monitoring TRP expression levels could provide a prognostic marker for identifying this insidious disease.

\section{Conclusion}

Ocular TRP functional expression is essential for mediating both adaptive and maladaptive responses to a wide variety of different environmental conditions that can impact tissue homeostasis maintenance. Table 1 summarizes ocular tissue TRP channel subtype localization and pharmacology described in pertinent references. As there is an association between aberrant TRP channel expression and human disease, these regulators of $\mathrm{Ca}^{2+}$ influx may be potential drug targets for usage in a clinical setting. Nevertheless, much effort is still needed to validate this suggestion.

Firstly, specific sites on TRP channel structure must be delineated that account for each of the responses mediated by selective channel activity modulation. Secondly, site-specific mutations must be expressed to determine if such alterations correspond with any of the pathophysiological responses by cells that express TRP channels. Finally, drug-induced reversal of any changes caused by disease must be selective without serious side effects. For example, even though TRPV1 inhibition by $\mathrm{CPZ}$ can be selective in some settings, this antagonist also elevates body temperature at the same time that it reduces an inflammatory response in some tissues. Accordingly, TRPV1 antagonist development to reduce fibrosis and the likelihood of chronic inflammation may require designing drugs interacting only with specific domains on TRPV1 channels linked to signalling pathways mediating these sight-compromising responses rather than those eliciting body temperature control. As described above, resolving site-specific regions on TRPV1 channels controlling different responses could be beneficial for fine tuning specific responses promoting reepithelialization rather than those inducing corneal opacification and neovascularization. Judging from the burgeoning number of publications per year, TRP drug targeting may yet improve management in a clinical setting of a of host diseases.

\section{Abbreviations}

TRPA: Transient receptor potential ankyrin; TRPC: Transient receptor potential canonical; TRPM: Transient receptor potential melastatin; TRPML: Transient receptor potential mucolipin; TRPN: Transient receptor potential no mechano-potential; TRPP: Transient receptor potential polycystin; TRPV: Transient receptor potential vanilloid.

\section{Competing interests}

The authors declare that they have no competing interests.

\section{Authors' contributions}

PSR performed literature searches and wrote drafts with the help of SM who also generated the summary table. WC checked all of the cited references to assure that they are correct and inserted in the proper places. All authors read and approved the final manuscript.

\section{Acknowledgements}

Stefan Mergler is supported by DFG (Me 1706/14-1) about a TRP channel related research project and received a grant from the DFG priority program 1629 ThyroidTransAct (Me 1706/13-1).

\section{Author details}

'School of Ophthalmology and Optometry, Wenzhou Medical University, Wenzhou, Zhejiang 325027, P.R. China. ${ }^{2}$ Charité-Universitätsmedizin Berlin, Campus Virchow-Klinikum, Klinik für Augenheilkunde, Augustenburger Platz 1, D-13353 Berlin, Germany.

Received: 21 November 2014 Accepted: 15 February 2015

Published online: 02 March 2015

\section{References}

1. Ryskamp DA, Redmon S, Jo AO, Krizaj D. TRPV1 and Endocannabinoids: Emerging Molecular Signals that Modulate Mammalian Vision. Cells. 2014;3:914-38.

2. Ramsey IS, Delling M, Clapham DE. An introduction to TRP channels. Annu Rev Physiol. 2006;68:619-47.

3. Sappington RM, Sidorova T, Long DJ, Calkins DJ. TRPV1: contribution to retinal ganglion cell apoptosis and increased intracellular Ca2+ with exposure to hydrostatic pressure. Invest Ophthalmol Vis Sci. 2009:50:717-28.

4. Weitlauf C, Ward NJ, Lambert WS, Sidorova TN, Ho KW, Sappington RM, et al. Short-term increases in transient receptor potential vanilloid-1 mediate stress-induced enhancement of neuronal excitation. J Neurosci. 2014;34:15369-81.

5. Okada Y, Reinach PS, Shirai K, Kitano A, Kao WW, Flanders KC, et al. TRPV1 involvement in inflammatory tissue fibrosis in mice. Am J Pathol. 2011;178:2654-64.

6. Ward NJ, Ho KW, Lambert WS, Weitlauf C, Calkins DJ. Absence of transient receptor potential vanilloid-1 accelerates stress-induced axonopathy in the optic projection. J Neurosci. 2014;34:3161-70. 
7. Cosens DJ, Manning A. Abnormal electroretinogram from a Drosophila mutant. Nature. 1969;224:285-7.

8. Caterina MJ, Schumacher MA, Tominaga M, Rosen TA, Levine JD, Julius D. The capsaicin receptor: a heat-activated ion channel in the pain pathway. Nature. 1997;389:816-24.

9. Song MY, Yuan JX. Introduction to TRP channels: structure, function, and regulation. Adv Exp Med Biol. 2010;661:99-108.

10. Latorre R, Zaelzer C, Brauchi S. Structure-functional intimacies of transient receptor potential channels. Q Rev Biophys. 2009;42:201-46.

11. Venkatachalam K, Montell C. TRP channels. Annu Rev Biochem. 2007;76:387-417.

12. Hardie RC. Photosensitive TRPs. Handb Exp Pharmacol. 2014;223:795-826.

13. Nilius B, Owsianik G, Voets T, Peters JA. Transient receptor potential cation channels in disease. Physiol Rev. 2007:87:165-217.

14. Nilius B, Owsianik G. Transient receptor potential channelopathies. Pflugers Arch. 2010;460:437-50

15. Schonherr R. Clinical relevance of ion channels for diagnosis and therapy of cancer. J Membr Biol. 2005;205:175-84.

16. O'Neil RG, Heller S. The mechanosensitive nature of TRPV channels. Pflugers Arch. 2005;451:193-203.

17. Plant TD, Strotmann R. Trpv4. Handb Exp Pharmacol. 2007;179:189-205.

18. Wu L, Gao X, Brown RC, Heller S, O'Neil RG. Dual role of the TRPV4 channel as a sensor of flow and osmolality in renal epithelial cells. Am J Physiol Renal Physiol. 2007;293:F1699-713.

19. McKemy DD, Neuhausser WM, Julius D. Identification of a cold receptor reveals a general role for TRP channels in thermosensation. Nature. 2002;416:52-8.

20. Mogrich A, Hwang SW, Earley TJ, Petrus MJ, Murray AN, Spencer KS, et al. Impaired thermosensation in mice lacking TRPV3, a heat and camphor sensor in the skin. Science. 2005:307:1468-72.

21. Sherkheli MA, Vogt-Eisele AK, Weber K, Hatt H. Camphor modulates TRPV3 cation channels activity by interacting with critical pore-region cysteine residues. Pak J Pharm Sci. 2013;26:431-8.

22. Voets T, Nilius B, Hoefs S, van der Kemp AW, Droogmans G, Bindels RJ, et al. TRPM6 forms the Mg2+ influx channel involved in intestinal and renal Mg2 + absorption. J Biol Chem. 2004;279:19-25.

23. Zurborg S, Yurgionas B, Jira JA, Caspani O, Heppenstall PA. Direct activation of the ion channel TRPA1 by Ca2+. Nat Neurosci. 2007;10:277-9.

24. Stone LS, Molliver DC. In search of analgesia: emerging roles of GPCRs in pain. Mol Interv. 2009;9:234-51.

25. Basbaum Al, Bautista DM, Scherrer G, Julius D. Cellular and molecular mechanisms of pain. Cell. 2009;139:267-84.

26. Bautista DM, Wilson SR, Hoon MA. Why we scratch an itch: the molecules, cells and circuits of itch. Nat Neurosci. 2014;17:175-82.

27. Grace PM, Hutchinson MR, Maier SF, Watkins LR. Pathological pain and the neuroimmune interface. Nat Rev Immunol. 2014;14:217-31.

28. Morales-Lazaro SL, Simon SA, Rosenbaum T. The role of endogenous molecules in modulating pain through transient receptor potential vanilloid 1 (TRPV1). J Physiol. 2013;591:3109-21.

29. Tiruppathi C, Minshall RD, Paria BC, Vogel SM, Malik AB. Role of Ca2+ signaling in the regulation of endothelial permeability. Vascul Pharmacol. 2002;39:173-85.

30. Gordon-Shaag A, Zagotta WN, Gordon SE. Mechanism of Ca(2+)-dependent desensitization in TRP channels. Channels (Austin). 2008:2:125-9.

31. Voets T. Quantifying and modeling the temperature-dependent gating of TRP channels. Rev Physiol Biochem Pharmacol. 2012;162:91-119.

32. Bautista DM, Siemens J, Glazer JM, Tsuruda PR, Basbaum Al, Stucky CL, et al. The menthol receptor TRPM8 is the principal detector of environmental cold. Nature. 2007:448:204-8.

33. McKemy DD. How cold is it? TRPM 8 and TRPA 1 in the molecular logic of cold sensation. Mol Pain. 2005;1:16.

34. McKemy DD. The molecular and cellular basis of cold sensation. ACS Chem Neurosci. 2013;4:238-47.

35. Tominaga M, Caterina MJ. Thermosensation and pain. J Neurobiol. 2004;61:3-12.

36. Zhong L, Bellemer A, Yan H, Ken H, Jessica R, Hwang RY, et al. Thermosensory and nonthermosensory isoforms of Drosophila melanogaster TRPA1 reveal heat-sensor domains of a thermoTRP Channel. Cell Rep. 2012;1:43-55.

37. Voets T, Droogmans G, Wissenbach U, Janssens A, Flockerzi V, Nilius B. The principle of temperature-dependent gating in cold- and heat-sensitive TRP channels. Nature. 2004;430:748-54.
38. Kraft $R$, Harteneck $C$. The mammalian melastatin-related transient receptor potential cation channels: an overview. Pflugers Arch. 2005:451:204-11.

39. Frederick J, Buck ME, Matson DJ, Cortright DN. Increased TRPA1, TRPM8, and TRPV2 expression in dorsal root ganglia by nerve injury. Biochem Biophys Res Commun. 2007;358:1058-64.

40. Klooster J, Blokker J, Ten Brink JB, Unmehopa U, Fluiter K, Bergen AA, et al. Ultrastructural localization and expression of TRPM1 in the human retina. Invest Ophthalmol Vis Sci. 2011;52:8356-62.

41. Shen Y, Heimel JA, Kamermans M, Peachey NS, Gregg RG, Nawy S. A transient receptor potential-like channel mediates synaptic transmission in rod bipolar cells. J Neurosci. 2009;29:6088-93.

42. Xu Y, Dhingra A, Fina ME, Koike C, Furukawa T, Vardi N. mGluR6 deletion renders the TRPM1 channel in retina inactive. J Neurophysiol. 2012;107:948-57.

43. Ryskamp DA, Witkovsky P, Barabas P, Huang W, Koehler C, Akimov NP, et al. The polymodal ion channel transient receptor potential vanilloid 4 modulates calcium flux, spiking rate, and apoptosis of mouse retinal ganglion cells. J Neurosci. 2011;31:7089-101.

44. Gilliam JC, Wensel TG. TRP channel gene expression in the mouse retina. Vision Res. 2011;51:2440-52.

45. Crousillac S, LeRouge M, Rankin M, Gleason E. Immunolocalization of TRPC channel subunits 1 and 4 in the chicken retina. Vis Neurosci. 2003;20:453-63.

46. Mergler S, Cheng Y, Skosyrski S, Garreis F, Pietrzak P, Kociok N, et al. Altered calcium regulation by thermosensitive transient receptor potential channels in etoposide-resistant WERI-Rb1 retinoblastoma cells. Exp Eye Res. 2012;94:157-73.

47. Pan Z, Yang H, Reinach PS. Transient receptor potential (TRP) gene superfamily encoding cation channels. Hum Genomics. 2011;5:108-16.

48. Cordeiro S, Seyler S, Stindl J, Milenkovic VM, Strauss O. Heat-sensitive TRPV channels in retinal pigment epithelial cells: regulation of VEGF-A secretion. Invest Ophthalmol Vis Sci. 2010;51:6001-8.

49. Mergler S, Derckx R, Reinach PS, Garreis F, Bohm A, Schmelzer L, et al. Calcium regulation by temperature-sensitive transient receptor potential channels in human uveal melanoma cells. Cell Signal. 2014;26:56-69.

50. Khajavi N, Reinach PS, Slavi N, Skrzypski M, Lucius A, Strauss O, et al. Thyronamine induces TRPM8 channel activation in human conjunctival epithelial cells. Cell Signal. 2015;27:315-25.

51. Mergler S, Garreis F, Sahlmuller M, Lyras EM, Reinach PS, Dwarakanath A, et al. Calcium regulation by thermo- and osmosensing transient receptor potential vanilloid channels (TRPVs) in human conjunctival epithelial cells. Histochem Cell Biol. 2012;137:743-61.

52. Kurose M, Meng ID. Dry eye modifies the thermal and menthol responses in rat corneal primary afferent cool cells. J Neurophysiol. 2013;110:495-504.

53. Murata Y, Masuko S. Peripheral and central distribution of TRPV1, substance P and CGRP of rat corneal neurons. Brain Res. 2006:1085:87-94.

54. Madrid R, Donovan-Rodriguez T, Meseguer V, Acosta MC, Belmonte C, Viana F. Contribution of TRPM8 channels to cold transduction in primary sensory neurons and peripheral nerve terminals. J Neurosci. 2006;26:12512-25.

55. Robbins A, Kurose M, Winterson BJ, Meng ID. Menthol activation of corneal cool cells induces TRPM8-mediated lacrimation but not nociceptive responses in rodents. Invest Ophthalmol Vis Sci. 2012;53:7034-42.

56. Bates BD, Mitchell K, Keller JM, Chan CC, Swaim WD, Yaskovich R, et al. Prolonged analgesic response of cornea to topical resiniferatoxin, a potent TRPV1 agonist. Pain. 2010;149:522-8.

57. Parra A, Madrid R, Echevarria D, del Olmo S, Morenilla-Palao C, Acosta MC et al. Ocular surface wetness is regulated by TRPM8-dependent cold thermoreceptors of the cornea. Nat Med. 2010;16:1396-9.

58. Sumioka T, Okada Y, Reinach PS, Shirai K, Miyajima M, Yamanaka O, et al Impairment of corneal epithelial wound healing in a TRPV1-deficient mouse. Invest Ophthalmol Vis Sci. 2014;55:3295-302.

59. Zhang F, Yang H, Wang Z, Mergler S, Liu H, Kawakita T, et al. Transient receptor potential vanilloid 1 activation induces inflammatory cytokine release in corneal epithelium through MAPK signaling. J Cell Physiol. 2007:213:730-9.

60. Mergler S, Garreis F, Sahlmuller M, Reinach PS, Paulsen F, Pleyer U. Thermosensitive transient receptor potential channels in human corneal epithelial cells. J Cell Physiol. 2011;226:1828-42.

61. Yang H, Mergler S, Sun X, Wang Z, Lu L, Bonanno JA, et al. TRPC4 knockdown suppresses epidermal growth factor-induced store-operated channel activation and growth in human corneal epithelial cells. J Biol Chem. 2005;280:32230-7

62. Yang H, Wang Z, Capo-Aponte JE, Zhang F, Pan Z, Reinach PS. Epidermal growth factor receptor transactivation by the cannabinoid receptor (CB1) 
and transient receptor potential vanilloid 1 (TRPV1) induces differential responses in corneal epithelial cells. Exp Eye Res. 2010;91:462-71.

63. Pan Z, Yang H, Mergler S, Liu H, Tachado SD, Zhang F, et al. Dependence of regulatory volume decrease on transient receptor potential vanilloid 4 (TRPV4) expression in human corneal epithelial cells. Cell Calcium. 2008;44:374-85.

64. Pan Z, Wang Z, Yang H, Zhang F, Reinach PS. TRPV1 activation is required for hypertonicity-stimulated inflammatory cytokine release in human corneal epithelial cells. Invest Ophthalmol Vis Sci. 2011;52:485-93.

65. Hua X, Su Z, Deng R, Lin J, Li DQ, Pflugfelder SC. Effects of I-Carnitine, Erythritol and Betaine on Pro-inflammatory Markers in Primary Human Corneal Epithelial Cells Exposed to Hyperosmotic Stress. Curr Eye Res. 2014;1:1-11.

66. Yang $Y$, Yang $H$, Wang Z, Mergler S, Wolosin JM, Reinach PS. Functional TRPV1 expression in human corneal fibroblasts. Exp Eye Res. 2013;107:121-9.

67. Yang Y, Wang Z, Yang H, Wang L, Gillespie SR, Wolosin JM, et al. TRPV potentiates TGFbeta-induction of corneal myofibroblast development through an oxidative stress-mediated p38-SMAD2 signaling loop. PLoS One. 2013;8:e77300

68. Marfurt CF, Cox J, Deek S, Dvorscak L. Anatomy of the human corneal innervation. Exp Eye Res. 2010;90:478-92.

69. Rae JL, Watsky MA. Ionic channels in corneal endothelium. Am J Physiol. 1996:270:C975-89.

70. Mergler S, Valtink M, Taetz K, Sahlmuller M, Fels G, Reinach PS, et al. Characterization of transient receptor potential vanilloid channel 4 (TRPV4) in human corneal endothelial cells. Exp Eye Res. 2011;93:710-9.

71. Mergler S, Valtink M, Coulson-Thomas VJ, Lindemann D, Reinach PS, Engelmann $K$, et al. TRPV channels mediate temperature-sensing in human corneal endothelial cells. Exp Eye Res. 2010;90:758-70.

72. Mergler S, Mertens C, Valtink M, Reinach PS, Szekely VC, Slavi N, et al. Functional significance of thermosensitive transient receptor potential melastatin channel 8 (TRPM8) expression in immortalized human corneal endothelial cells. Exp Eye Res. 2013;116:337-49.

73. Hsu JK, Cavanagh HD, Jester JV, Ma L, Petroll WM. Changes in cornea endothelial apical junctional protein organization after corneal cold storage. Cornea. 1999;18:712-20.

74. Lindstrom RL. Advances in corneal preservation. Trans Am Ophthalmol Soc. 1990;88:555-648.

75. Mergler S, Pleyer U, Reinach P, Bednarz J, Dannowski H, Engelmann K, et al. EGF suppresses hydrogen peroxide induced $\mathrm{Ca} 2+$ influx by inhibiting L-type channel activity in cultured human corneal endothelial cells. Exp Eye Res. 2005:80:285-93.

76. Shahidullah M, Mandal A, Delamere NA. TRPV4 in porcine lens epithelium regulates hemichannel-mediated ATP release and Na-K-ATPase activity. Am J Physiol Cell Physiol. 2012;302:C1751-61.

77. Martinez-Garcia MC, Martinez T, Paneda C, Gallego P, Jimenez Al, Merayo J. Differential expression and localization of transient receptor potential vanilloid 1 in rabbit and human eyes. Histol Histopathol. 2013;28:1507-16.

78. Kaneko Y, Szallasi A. Transient receptor potential (TRP) channels: a clinical perspective. Br J Pharmacol. 2014;171:2474-507.

79. Bach G. Mucolipin 1: endocytosis and cation channel-a review. Pflugers Arch. 2005:451:313-7.

80. Irie S, Furukawa T. Trpm1. Handb Exp Pharmacol. 2014;222:387-402.

81. Islam MS. TRP channels of islets. Adv Exp Med Biol. 2011;704:811-30.

82. Jacobson DA, Philipson LH. TRP channels of the pancreatic beta cell. Handb Exp Pharmacol. 2007;179:409-24.

83. Morita $Y$, Kimura K, Fujitsu Y, Enomoto A, Ueno S, Kondo M, et al. Autoantibodies to transient receptor potential cation channel, subfamily M, member 1 in a Japanese patient with melanoma-associated retinopathy. Jpn J Ophthalmol. 2014;58:166-71.

84. Ueno S, Ito $\mathrm{Y}$, Maruko R, Kondo M, Terasaki H. Choroidal atrophy in a patient with paraneoplastic retinopathy and anti-TRPM1 antibody. Clin Ophthalmol. 2014;8:369-73.

85. Tran VT, Ho PT, Cabrera L, Torres JE, Bhattacharya SK. Mechanotransduction channels of the trabecular meshwork. Curr Eye Res. 2014;39:291-303.

86. Kuck JF, Kuck KD. The Emory mouse cataract: increased accumulation of calcium during cataractogenesis. Lens Eye Toxic Res. 1989;6:853-62.

87. Chang AE, Karnell LH, Menck HR. The National Cancer Data Base report on cutaneous and noncutaneous melanoma: a summary of 84,836 cases from the past decade. The American College of Surgeons Commission on Cancer and the American Cancer Society. Cancer. 1998;83:1664-78.

88. Singh AD, Turell ME, Topham AK. Uveal melanoma: trends in incidence, treatment, and survival. Ophthalmology. 2011;118:1881-5.
89. Devi S, Kedlaya R, Maddodi N, Bhat KM, Weber CS, Valdivia H, et al. Calcium homeostasis in human melanocytes: role of transient receptor potential melastatin 1 (TRPM1) and its regulation by ultraviolet light. Am J Physiol Cell Physiol. 2009;297:C679-87.

90. Yamamura H, Ugawa S, Ueda T, Morita A, Shimada S. TRPM8 activation suppresses cellular viability in human melanoma. Am J Physiol Cell Physiol. 2008;295:C296-301.

91. Lohmann D. Retinoblastoma. Adv Exp Med Biol. 2010;685:220-7.

92. Hanano T, Hara Y, Shi J, Morita H, Umebayashi C, Mori E, et al. Involvement of TRPM7 in cell growth as a spontaneously activated Ca2+ entry pathway in human retinoblastoma cells. J Pharmacol Sci. 2004;95:403-19.

93. Yang $H$, Sun $X$, Wang Z, Ning G, Zhang F, Kong J, et al. EGF stimulates growth by enhancing capacitative calcium entry in corneal epithelial cells. J Membr Biol. 2003;194:47-58.

94. Leonelli M, Martins DO, Britto LR. TRPV1 receptors are involved in protein nitration and Muller cell reaction in the acutely axotomized rat retina. Exp Eye Res. 2010;91:755-68.

95. Yamada T, Ueda T, Ugawa S, Ishida Y, Imayasu M, Koyama S, et al. Functional expression of transient receptor potential vanilloid 3 (TRPV3) in corneal epithelial cells: involvement in thermosensation and wound healing. Exp Eye Res. 2010;90:121-9.

96. Hirata H, Oshinsky ML. Ocular dryness excites two classes of corneal afferent neurons implicated in basal tearing in rats: involvement of transient receptor potential channels. J Neurophysiol. 2012;107:1199-209.

\section{Submit your next manuscript to BioMed Central and take full advantage of:}

- Convenient online submission

- Thorough peer review

- No space constraints or color figure charges

- Immediate publication on acceptance

- Inclusion in PubMed, CAS, Scopus and Google Scholar

- Research which is freely available for redistribution

Submit your manuscript at www.biomedcentral.com/submit 